\title{
Enseigner les compétences interculturelles à l'ère du Covid 19, expérience $E$-tandems entre l'université Hassan 1er au Maroc et l'Université d'Angers en France
}

\author{
Teaching intercultural skills in the age of Covid 19, \\ E-tandems experience between Hassan 1st University \\ in Morocco and Angers University in France
}

\author{
Raj Mohammed \\ Université Hassan $1^{\text {er- }}$ Settat-Maroc \\ mohammed.raj@uhp.ac.ma
}

\begin{abstract}
To ensure educational continuity which has been broken by the Coronavirus, Hassan 1 University in Morocco and the University of Angers in France have pooled an E-tandem project for teaching French as a foreign language (FLE). On the Angevin side, the experience will allow students of the FLE didactics to carry out their internship with an audience belonging to different geographic and cultural backgrounds; on the Moroccan side, eighteen students from two different schools belonging to Hassan 1 University have been chosen to live opening moments to develop their linguistic and communicative skills with French natives. The tandems are designed based on the needs of Moroccan students with a thematic program concerted by the educational managers of the two universities. The intercultural dimension will be the foundation of this experience, the main aim of which is to use new technologies to understand the Others as far as their linguistic, cultural and civilizational aspects.

Keywords

e-tandems, intercultural, skills, inter-comprehension.
\end{abstract}

\begin{abstract}
Resumen
Para garantizar la continuidad educativa interrumpida por el coronavirus, la Universidad Hassan 1 en Marruecos y la Universidad de Angers en Francia y agrupar un proyecto de tándem electrónico para enseñar francés como lengua extranjera (FLE). Del lado angevino, La experiencia permitirá a los estudiantes de las didácticas FLE realizar su pasantía con una audiencia que pertenece a diferentes entornos geográficos y culturales; en el lado marroquí, estudiantes de Hassan 1 Universidad dieciocho estudiantes elegidos en dos establecimientos, vivirá en momentos de apertura para desarrollar sus habilidades lingüísticas y comunicativas con los nativos franceses. Los tándems están diseñados en función de las necesidades de los estudiantes marroquíes con un programa temático concertado por los gerentes educativos de las dos universidades. La dimensión intercultural será la base de esta experiencia, cuyo objetivo principal es utilizar nuevas tecnologías para comprender al Otro en sus aspectos lingüísticos, culturales y de civilización.

Palabras clave

e-tandems, habilidades, interculturales, intercomprensión.
\end{abstract}




\section{Introduction}

"Pour étudier l'homme, il faut apprendre à porter sa vue au loin" disait Jean Jacques Rousseau dans Essai sur l'origine des langues. Telle était l'objectif principal de l'expérience E-tandem de l'Université d'Angers en France et de 1'Université Hassan $1^{\text {er }}$ au Maroc. Ce projet initié par les collègues français en janvier 2021,dans le contexte du Coronavirus qui a profondément lesté, voire paralysé le système éducatif à l'échelle universelle. Le projet en question a été conçu pour être opérationnalisé en ligne durant une période de trois mois. En effet, apprendre à un étudiant de voir par-delà les frontières géographiques, linguistiques et culturelles c'est lui offrir l'occasion et la chance de mieux se définir dans ce vaste monde qui se connait et s'ignore dans une logique fortement gouvernée plus par les décisions politiques que par les soucis pédagogiques. Parmi les outils de l'intercompréhension il y a le fait linguistique, car apprendre une langue, c'est permettre à d'autres cultures de contribuer à former notre identité culturelle. Les mots que nous utilisons aujourd'hui pour échanger sur les affaires de la vie quotidienne nous proviennent de différents "ruissellements" et formant un "bassin sémantique" dont la complexité incite parfois à la rêverie'. Le présent travail ne prétend pas présenter une étude théorique approfondie sur la question de l'interculturel en classe de français langue étrangère, mais vise à donner un aperçu d'une expérience vécue par les étudiants de l'Institut des sciences du sport et ceux de l'Institut des sciences de la Santé dans le cadre d'un partenariat avec l'université d'Angers en France et l'université Hassan1er au Maroc.

Trois parties constituent la charpente de cet article:

- la première concerne l'organisation du projet E-tandem;

- la deuxième partie traite de la mise en route de l'expérience;

- le troisième et le dernier point est consacré à la construction du discours interculturel lors des échanges en classe de français langue étrangère.

\subsection{Organisation de l'expérience}

Pour accompagner ces étudiants dans ce projet, nous avons commencé par prendre en charge le cours de langue et de terminologie programmé au premier semestre pour être proche de toutes les étapes de l'expérience. Un autre collègue s'est chargé de faire le suivi durant le deuxième semestre. L'enveloppe horaire impartie à cet élément du module est de 25 heures. Ce contact nous a permis de constituer une idée claire sur le niveau de langue de ces étudiants qui ont passé presque la moitié de l'année à étudier en ligne avec toute la complexité de l'entreprise. Psychologiquement, ces étudiants même en présentiel ne semblent

1 Nous employons la notion de "ruissellement" et celle de "bassin sémantique" dans le sens que leur donne Gibert Durand dans son ouvrage Introduction à la mythodologie, Tunis, Cérès-éditions, 1996. 
pas sortir des réflexes du confinement qui n'a laissé chez eux que peur, incertitude, confusion et perturbation des idées.

L'opération E-tandem était pour ses étudiants une occasion de sortir de l'ambiance de la pandémie pour s'ouvrir sur une expérience unique qui va leur permettre de découvrir d'autres horizons linguistiques et culturels. Quatre tandems, formés chacun de trois étudiants, doivent composer avec un professeur stagiaire durant deux mois en ligne en utilisant meet Google et/ou WhatsApp. La séance dure, en générale, une heure jusqu'à une heure et demie.

En concertation avec l'équipe angevine, nous avons choisi comme objectif majeur à cette expérience la compréhension et la production de l'oral. Dans une démarche contractuelle, les étudiants ont accepté à l'unanimité d'adhérer à ce projet vu leurs lacunes à l'oral. La méthode pédagogique utilisée par excellence et la classe inversée. Bien évidemment, lorsqu'il s'agit d'une classe de FLE, il est très difficile de privilégier une approche pédagogique par rapport à une autre. Nous estimons qu'une compétence ne saurait être développée chez un apprenant que par une approche didactique complexe. Ainsi, le fait de croiser la pédagogie par objectifs avec l'approche par les compétences, la classe inversée, la perspective actionnelle augmenterait les chances d'aboutir à des résultats probants étant donné la différence dans les capacités de réception chez les apprenants surtout que ces derniers n'apprennent pas tous de la même façon, avec les mêmes motivations et les mêmes besoins. Didactiser une séance de cours c'est concevoir une méthode de transmission de messages adaptée à chaque apprenant en fonction de ses capacités mentales et cognitives. Ci-après un tableau résumant le projet:

\begin{tabular}{ll}
\hline Etablissements impliqués dans le projet & $\begin{array}{l}\text { L'institut des sciences du sport } \\
\text { L'institut supérieur des Sciences de la santé }\end{array}$ \\
\hline
\end{tabular}

Institut Supérieur des sciences de la santé 1 tandem de 6 étudiants (I3S)

\begin{tabular}{ll}
\hline Institut des sciences du sport (I2S) & 4 tandems de 3 étudiants chacun \\
\hline Compétences ciblées & Comprendre l'oral/Produire l'oral \\
\hline Durée des tandems & Du 15/2/21 au 15/5/2021 \\
\hline Approche didactique choisie & La classe inversée \\
\hline Moyens utilisés & Meet google-WhatsApp \\
\hline
\end{tabular}

À fortiori, les tandems fonctionnent selon une logique participative. En effet, l'étudiant n'est pas devant un professeur "sait tout" et un étudiant qui ne sait rien; c'est l'inverse qui se produit dans une situation pédagogique ou l'enseignant adopte la posture de guide, d'encadrant et d'auditeur attentif au discours construit par l'étudiant. Selon le principe de la classe inversée, l'étudiant effectue une recherche prend des notes, les organise dans un plan et les présente oralement devant ses camarades. Il s'agit ici de trois capacités à opérationnaliser: lire comprendre et s'exprimer. 
En termes actionnels, l'étudiant doit réaliser une tâche finale à savoir la production d'un discours qui peut se présenter sous forme de récit de voyage, de description d'un lieu, d'une information sur un phénomène ou un thème précis ou une explication d'une situation de la vie quotidienne.

\subsection{Mise en route des E-tandems}

Trois réunions ont été organisées avant de commencer l'opération tandem:

- Une réunion en ligne avec la coordonnatrice du projet: Angers/France.

- Une réunion avec les stagiaires et les tuteurs marocains pour définir les besoins des étudiants marocains en matière du FLE, les objectifs à atteindre et les modalités de travail et d'évaluation.

- Une dernière réunion en ligne avec tous les participants (stagiaires, tuteurs et étudiant-e-s).

Cette réunion élargie était l'occasion de rassembler tous les étudiant-e-s participant au projet $E$-tandem pour rappeler les éléments du contrat pédagogique. Dans ce genre d'action institutionnelle, l'étudiant doit comprendre qu'il n'est pas obligé de participer à cette expérience s'il n'en a pas envie et que, avant le démarrage des séances, il a toujours le temps de se retirer si cela ne convient ni à son agenda ni à ses besoins en matière de l'oral. C'est pour cette raison que nous avons pensé à établir un contrat pédagogique pour montrer, d'une part, l'aspect sérieux de l'affaire, et inciter, d'autre part, les étudiants à prendre conscience de l'ampleur de l'action et, partant, être responsables de leur choix et décisions.

Le contrat établi a mis l'accent sur les principes classiques du contrat pédagogique, à savoir: le consentement mutuel, l'acceptation positive de l'apprenant, la négociation de tous les éléments du contrat, l'engagement réciproque. Cette étape de métacommunication se révèle constructive parce qu'elle définit les rôles de chaque étudiant participant et prévoit les obstacles et les remédiations en même temps. C'est après cette étape que le processus commence selon la démarche qualité: on part d'une situation réelle diagnostiquée, avec des données sur le niveau linguistique des étudiants, on prévoit un programme de formation et des outils de régulation afin d'aboutir aux résultats escomptés. 


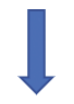

situation réelle

Résultat de

l'évaluation

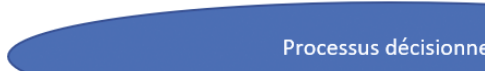

1

I

Processus

souhaitée

Régulations et

réajustement durant les
Situation

Résultats escomptés

\section{Tandems et construction de la compétence interculturelle}

\section{1.Contexte général: la question identitaire dans le rapport Nord/Sud}

Actuellement, le monde connait des changements profonds et rapides dus aux instabilités des systèmes politiques et aux effets directs de la mondialisation. De ce fait, les populations des régions défavorisées se mettent sur les routes à la quête d'un Eldorado, d'une terre d'accueil où il serait possible de vivre avec dignité et respect mutuel des différences ethniques. En Occident, beaucoup d'immigrés ont fondé une famille et se sont insérés dans le tissu économique du pays d'accueil. Cependant, la confrontation avec de nouvelles cultures peut être source de confort ou de malaise social selon que le pays d'accueil respecte la différence et reconnait à l'étranger ses droits et devoirs, ou que, au contraire, il tente d' "intégrer" l'immigré, mais dans un processus d'assimilation pour ne pas dire aliénation.

Aujourd'hui, le Maroc qui était pendant les dernières décennies du siècle passé, un pays de transit, de passage pour les migrants, devient un carrefour migratoire et un pays d'accueil. D'où l'intérêt du développement de la traduction comme outil de connaissance de l'autre et comme vecteur de culture et d'interculturalité. En effet, à la fin 2014, le Maroc a signé de nombreux accords bilatéraux avec des États d'Afrique de l'Ouest (Mali, Sénégal, Côte d'Ivoire, Gabon ...). Ce "marketing diplomatique" a pour but de consolider la position géostratégique du Maroc, mais par-delà les considérations socioéconomiques et sociopolitiques, le Maroc, en affirmant son africanité, devrait faire face à une autre réalité sociétale et prévoir un plan stratégique mettant en exergue toutes les questions culturelles, cultuelles, linguistiques et identitaires. Les mobilités étudiantes qui vont grandissantes dans établissements scolaires et universitaires publics et privés, la "mobilité religieuse" instaurée par le Roi du Maroc pour la formation d'Imams africains, les retraités étrangers qui se sont installés au Maroc selon les saisons ou pour toute l'année, ces différentes composantes humaines font du Maroc une 
mosaïque ethnique et culturelle qui exige une étude scientifique dûment élaborée avec des concepts-clés faisant le tour des toutes les disciplines des sciences humaines et sociales.

C'est dans cette réflexion de type anthropologique que s'inscrit le projet E-tandem pour assurer la continuité de l'apport du Cadre européen commun de référence pour les langues qui a accordé une importance particulière à des notions clés telles que "plurilinguisme", "pluriculturalisme" et "compétence plurilingue et pluriculturelle". Dans la réalité, ces concepts expliquent plus ce qu'il faudrait faire que ce qui se fait réellement dans un contexte marqué par le cloisonnement culturel. Un nouveau paradigme émerge faisant de la perspective interculturelle un exercice de citoyenneté impliquant l'humanité entière pour vivre dans la différence.

\title{
2.2. Catégories universelles choisies et co-élaboration du discours interculturel
}

D'une manière générale, chaque culture est de nature interculturelle. Il n'y a qu'à consulter un dictionnaire de langue pour s'apercevoir de la quantité d'emprunts et de ces mots voyageurs, traversant siècles et frontières géographiques pour enrichir notre langue. Notre cuisine aussi témoigne de la richesse des produits alimentaires provenant de sol géographique et culturel différents. Dans le cadre de l'apprentissage d'une langue, l'apprenant tante d'imiter la langue cible des natifs, mais lorsqu'il s'agit des affaires culturelles, il n'est nullement question de substituer la culture cible à la culture source:

\begin{abstract}
Alors que l'apprenant cherche à imiter le mieux possible la langue parlée par les natifs (prononciation, registres, tournure...), l'enseignement de la culture, lui, ne vise pas l'adoption pure et simple des divers aspects de la culture- cible, mais relèvent de divers interprétations et transactions. (Defays, 2016: 152-153)
\end{abstract}

Une autre particularité de la perspective interculturelle, c'est que lorsqu'il s'agit d'enseigner une langue, généralement les contenus et les objectifs sont clairs et mesurables, mais dans le champ de l'interculturel, il n'y a pas de contenu à enseigner ni d'objectifs clairement définis auquel pourrait prétendre les interactions qui, elles, restent liées au degré de l'acceptation de l'autre. S'ajoute à cela que

toute rencontre interculturelle ou non nécessite une action conjointe. Cela signifie que personne ne peut être compétent au niveau interculturel en tant qu'individu: la compétence nécessite un effort coordonné de tous les participants à une interaction, puisque toute interaction est coconstruite. (Wendy Leeds- Hurwitz, 2016: 18)

Dans ce sens, l'approche interculturelle ne serait possible et opérationnelle que dans une démarche participative dépassant le niveau individuel vers la compréhension et la construction d'un dialogue interculturel. 
Durant les deux mois de l'expérience E-tandem, un ensemble de thèmes constituait l'armature des échanges interculturels entre les professeurs stagiaires et les étudiants. On peut citer comme exemples les axes suivants:

- le Slam

- les stéréotypes

- vivre en France et au Maroc

- le féminisme

- le voyage

- la notion de “week-end” chez l'étudiant français et l'étudiant marocain.

Selon le principe de base de la classe inversée, l'étudiant, suivant les directives de son professeur, effectue une recherche sur le thème à l'ordre du jour et prépare un exposé oral à visée généralement informative et/ou argumentative. Seulement, par-delà la compétence linguistique visée au départ, à savoir la production de l'oral, l'échange interculturel se construit dans un processus transactionnel en réajustement permanent parce que, à part la première rencontre des présentations, enseignants et étudiants interagissent pour la première fois et se découvrent les uns les autres à travers leurs représentations sur les axes de recherche choisis et à travers aussi leurs comportements et attitudes. En effet, si les usages et les références culturelles étaient partagés entre les étudiants marocains, tel n'était pas le cas pour les professeurs stagiaires françaises dont la plupart avait besoin de plus de temps pour comprendre quelques composantes de la culture marocaine dans son sens le plus large ${ }^{2}$.

D'un point de vue managérial, "Il importe de bien connaitre les usages et les codes culturels de ses interlocuteurs qui peuvent varier d'un pays ou groupe de pays à un autre" (MEIER, 2016: 143).

Par ailleurs, le projet E-tandem a fait l'objet d'une évaluation par le biais d'un questionnaire et d'une entrevue en ligne avec tous les acteurs des tandems. Les entrevues ont été planifiées selon les disponibilités des étudiants participants. L'objectif était d'entrer en interaction direct avec les apprenants pour avoir un retour sur leur expérience et surtout écouter leurs remarques et propositions susceptibles d'améliorer les tandems prévus pour l'année universitaire 2021/2022. L'investigation portait sur un double aspect: la compétence linguistique et communicative puisque c'était l'objectif majeur assigné à ce projet, et d'un autre côté les échanges interculturels qui se sont élaborées dans les moments d'apprentissage durant deux mois d'étude ${ }^{3}$.

Concernant le développement des compétences linguistiques et communicatives des

2 Avant le processus de la marocanisation des cadres de l'enseignement au Maroc dans les années quatre-vingt, la fréquence du contact des élèves avec les professeurs étrangers: français, portugais, espagnols qui enseignaient dans les collèges et lycées marocains a permis aux uns et aux autres de connaitre les codes et les usages qui formaient une mosaïque de cultures et de langue. Aujourd'hui, cet aspect a disparu; emportant ainsi les relations altéritaires naissant du contexte immédiat.

3 Les entrevues en ligne ont été dirigées par les coordonnateurs du projet Mohamed RAJ au Maroc et Maeva 
étudiants, l'expérience a permis à ces derniers de mettre en pratique leurs connaissances théoriques en matière de langue française (lexique, prononciation, tournures syntaxiques, tonalité) le travail dans un petit groupe les à encouragés à prendre la parole chaque séance pour présenter un exposé, discuter et donner leur point de vue sur le sujet traité. D'après les témoignages des étudiants, ce scénario du learning by doing, mettant l'apprenant dans une série de situations-problèmes semble efficace parce qu'ils se sentaient fortement impliqués dans le processus. Ils tentent de "mobiliser un ensemble de ressources cognitives (savoirs, capacité, informations, etc) pour faire face avec pertinence et avec efficacité à une famille de situations" (Perrenoud, 2000) auxquelles ils sont confrontés. L'approche par les compétences utilisée comme adjuvant de la pédagogie de la classe inversée favorise un enseignement-apprentissage contextualisé, ciblant des performances langagières, cognitive et pragmatique. Il est vrai que l'enseignement à distance s'inscrit dans la logique de la distanciation avec toutes ses implications pédagogiques et humaines, mais cela n'empêche pas l'apprenant d'effectuer des tâches et d'intégrer petit à petit d'autres modalités de travail qui, elles, ne devraient pas être obligatoirement similaires à celles pratiquées en présentiel. Cette dichotomie présentiel/ distanciel devient plus imposante et plus pertinente lorsqu'il s'agit d'aborder les aspects interculturels.

Pour ce qui est de l'aspect interculturel des E-tandems, les thématiques programmées par les enseignantes stagiaires ont donné lieu à des échanges instructifs et constructifs. Instructifs parce que chaque thème, objet de recherche et de discussion, était une occasion de partager des informations sur plusieurs aspects culturels en France et au Maroc. Ce partage d'informations relevant du vécu des participants a permis aux intervenants de comparer leurs représentations sur des catégories universelles touchant à l'existence humaine. On peut citer comme exemples les éléments suivants:

- Le slam, les étudiants marocains découvrent pour la première fois cette forme de poésie et tente de "slamer" pour développer leurs compétences linguistiques et communicatives.

- Louer un appartement en France: Les étudiants marocains ont été surpris par la complexité de l'entreprise relative à la location d'un appartement en France au cas où ils seraient tentés de continuer leurs études en France. De leur côté, les enseignantes stagiaires françaises ont remarqué la simplicité de l'opération au Maroc.

- Le week-end: l'échange a montré qu'un week-end pour un étudiant français correspond à un moment de repos de toute activité institutionnelle. L'étudiant peut vaquer à des préoccupations personnelles pour faire ce dont il a envie, loin des contraintes de l'école. Cet état de fait n'est pas partagé avec les étudiants marocains qui, la plupart du temps, profite du week-end pour étudier. de langue et communication à l'Institut des sciences du sport et l'institut supérieur des sciences de la santé. 
L'échange a conduit les étudiants à se renseigner sur la symbolique des jours de la semaine, en particulier "dimanche" pour comprendre que se reposer le week-end est un comportement qui plonge ses racines dans l'histoire de la religion chrétienne. Tel est le cas du jour "vendredi" où les musulmans doivent tout délaisser pour faire leur prière dont le rituel est différent des autres prières de la semaine.

- Ramadan4: le vocable "jeuner" existe dans toutes les cultures, mais s'abstenir de boire et de manger durant 14 heures a fait l'objet d'une discussion entre les étudiants et leurs enseignantes. L'enjeu est double car, d'une part, les apprenants sont amenés à s'exprimer pour améliorer leur niveau de langue, et d'autre part, ils se sentent responsables dans la mesure où ils doivent expliquer à leur professeur en quoi consiste "ramadan" d'une manière correcte et pertinente.

Ces exemples, entre autres, montrent l'importance de l'intégration de la perspective interculturel dans les formations universitaires en ligne et/ou en présentiel. Car tout échange interculturel est porteur de significations constructives au niveau des relations humaines qui, elles, ne sont pas exclusivement liées aux études mais au domaine professionnel aussi. Ce domaine exige qualités et performance.

Dans une telle perspective, la notion de qualité est centrée sur la construction, il n'y a pas de critère universel imposé d'emblée à tous les acteurs, on recherche plutôt à susciter la construction par les acteurs eux-mêmes d'un cadre de référence commun tout en tenant compte de leur diversité (Bonamy, 2007: 189).

\section{Conclusion}

Intégrer la perspective interculturelle dans le cursus de formation des étudiants est une nécessité dictée par les mutations sociales que connait le monde depuis déjà deux décennies. Aujourd'hui, les mobilités étudiantes devraient être appréhendées en termes de formations à l'interculturel pour, non seulement partager des informations ignorées de part et d'autre, mais construire un discours respectant la diversité et favorisant l'échange dans la différence. Dans le cas des tandems expérimentés par l'université d'Angers en France et l'université Hassan 1er au Maroc, ce projet était un point de départ d'une réflexion et d'une action à adopter et à améliorer pour permettre aux futurs étudiants participants de bénéficier de deux choses différentes mais complémentaires: apprendre à apprendre par le biais, entre autres méthodes, de la classe inversée et, en même temps, tirer profit des technologies de l'information et de la communication pour une meilleure gestion de la diversité culturelle

4 Ramadan est le neuvième mois du calendrier hégirien. Il est le seul mois dont le nom est cité dans le Coran. Un mois saint qui constitue le mois du jeûne. 
qui reste une dimension fondamentale dans l'approche qualité relative au networking. Il est de la responsabilité de l'université d'unir les esprits en dehors des frontières géographiques et culturelles. Il est de sa responsabilité aussi de mettre en place des dispositifs éducatifs et managériaux pour élaborer des offres pédagogiques qui mettent en perspective la question interculturelle dans sa dimension académique et humaine.

\section{Références bibliographiques}

Bonamy, Joêl. 2007. "La formation entre normalisation et innovation l'enjeu de la construction de la qualité" in Presses universitaires de Québec (coll. Education-Recherche), 74-82.

DefaYs, Jean-Marc. 2017. "Servitude et grandeur pédagogiques. Les nouvelles circonstances et exigences du métier de professeur de français langue étrangère" in Dialogues et cultures, $n^{\circ} 63,23-31$.

Mustapha, Bencheikh. 2009. "Littérature et altérité” in Littérature et altérité, OKAD, 27-41.

Mohamed, Raj. 2017. "La perspective actionnelle: genèse et expérimentation pédagogique" in Le Langage et l'Homme. Paris, L'Harmattan, 1-2.

Perrenoud, P. 1995. "Des savoirs aux compétences: les indices sur le métier d'enseignant et sur le métier d'élève" in Pédagogie collégiale. Quebec, vol. 9, nº 1, 54-72.

Wendy, Leeds-Hurwitz. 2016. "De la possession des compétences interculturelles au dialogue interculturel: un cadre conceptuel" in Les Politiques sociales, 3 et 4, 18. 


\section{Annexe 1}

Questionnaire d'évaluation de l'expérience E-Tandem 2020-2021.

Ce questionnaire vise à évaluer l'expérience E-tandem avec l'université d'Angers. Vos réponses et suggestions contribueront à porter un regard réflexif sur cette expérience afin d'opérer les bons choix pédagogiques en matière de FLE.

1.-Vous êtes de sexe
o $\mathrm{M}$
o $\mathrm{F}$

2.- Établissement
o $\quad$ I $2 \mathrm{~S}$
o ISSS

3.- Filière choisie
o MS
o EPS
o Sciences de la santé

4.- Avez-vous participé à l'expérience E-Tandem?
o OUI
o NON

5.- Avez-vous manqué des séances?
o Souvent
o Quelquefois
o Jamais

6.- Avez-vous manqué des séances parce que

o La programmation ne vous convient pas

o Les contenus des séances ne répondent pas à vos attentes

o Vous avez des problèmes techniques

o Vous n'avez pas à suivre le rythme

o Autres à préciser 
7.- Vous trouvez ces séances
o Intéressantes
o peu intéressantes
o Pas du tout intéressantes

8.- Quelles compétences avez-vous développées lors de ces séances?
o Compétence de l'écoute
o Compétence de la prise de parole
o Compétence interculturelle

9.- Avez- vous rencontré des problèmes à travailler en ligne?
o OUI
o Non

10.- Quels outils numérique avez-vous utilisés?
o Zoom
o meet google
o WhatsApp
o Teams
o Autres à préciser

11.- L'apprentissage du FLE (français langue étrangère) avec des encadrant(e)s français(e)s est:
o Efficace
o Peu efficace
o Pas du tout efficace

12.- Quelle est la dimension qui vous a interpellé dans cette expériences E-Tandem?
o La communication
o L'apprentissage de la langue
o La rencontre avec des professeure(s) français(e)s
o La différence de culture

13.- Qu'est-ce que vous appris de cette expérience au niveau culturel?

Pensez-vous que cette expérience est à
o À refaire
o À ne plus répéter 
Anales de Filología Francesa, n. ${ }^{\circ}$ 29, 2021

RAJ MOHAMMED

\section{Annexe 2}

Réponses des étudiants

Vous êtes de sexe

$8 \&$ nbsp;réponses

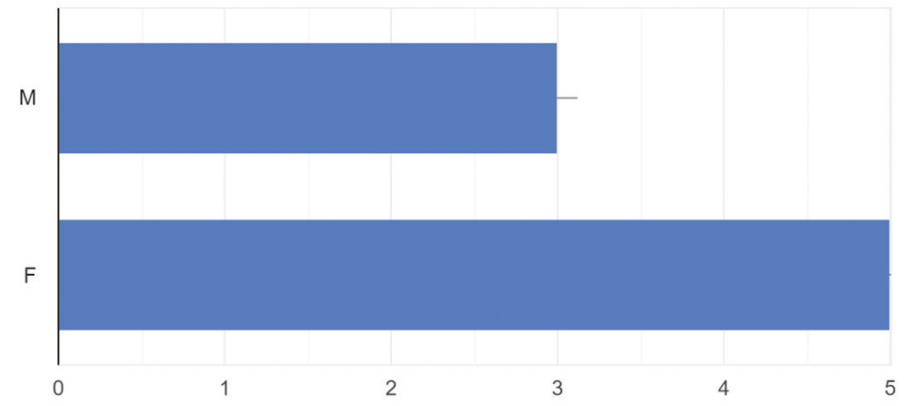

Etablissement

8\&nbsp;réponses

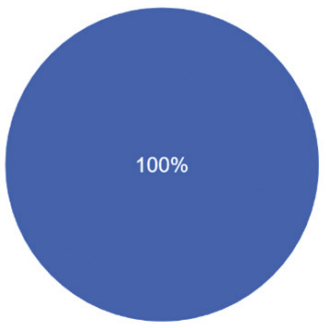

125

Isss

Filière

8\&nbsp;réponses

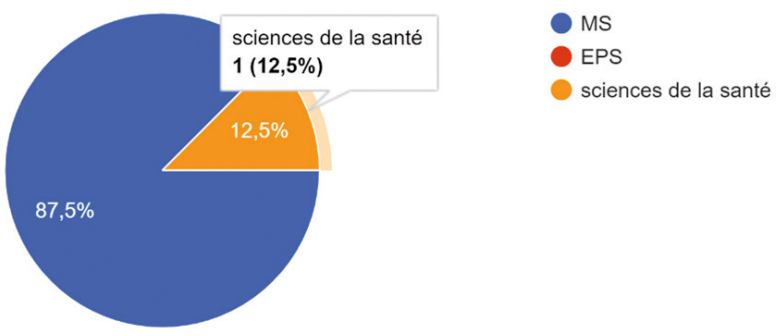


Anales de Filología Francesa, n. ${ }^{\circ}$ 29, 2021

ENSEIGNER LES COMPÉTENCES INTERCULTURELLES À L'ÈRE DU COVID 19, EXPÉRIENCE E-TANDEMS...

\section{Vous avez manqué des séances parce que}

4\&nbsp;réponses

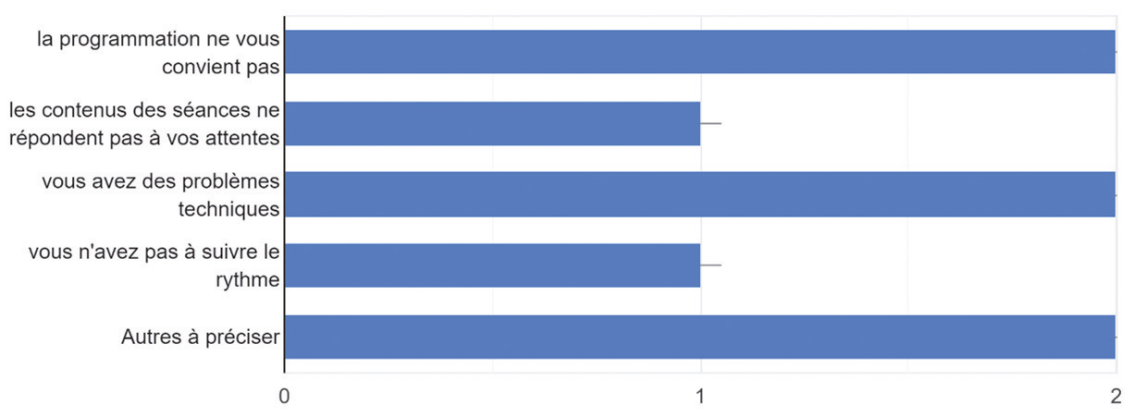

Vous trouvez ces séances

$8 \&$ \&bsp;réponses

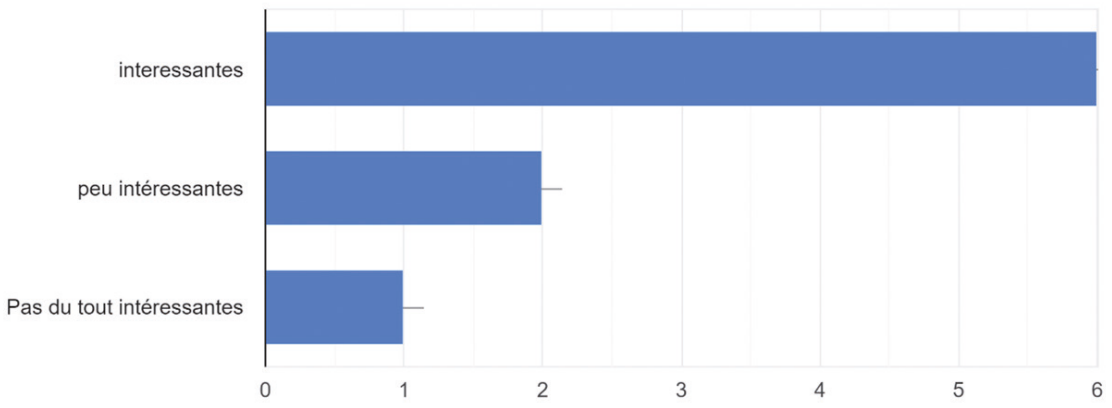


Anales de Filología Francesa, n. ${ }^{\circ}$ 29, 2021

RAJ MOHAMMED

Quelles compétences avez-vous développées lors de ces séances?

7\&nbsp;réponses

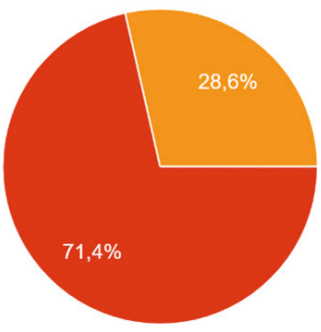

Compétence de l'écoute

compétence de la prise de parole

compétence interculturelle

L'apprentissage du FLE ( français langue étrangère) avec des encadrant(e)s français(e)s est 7\&nbsp;réponses

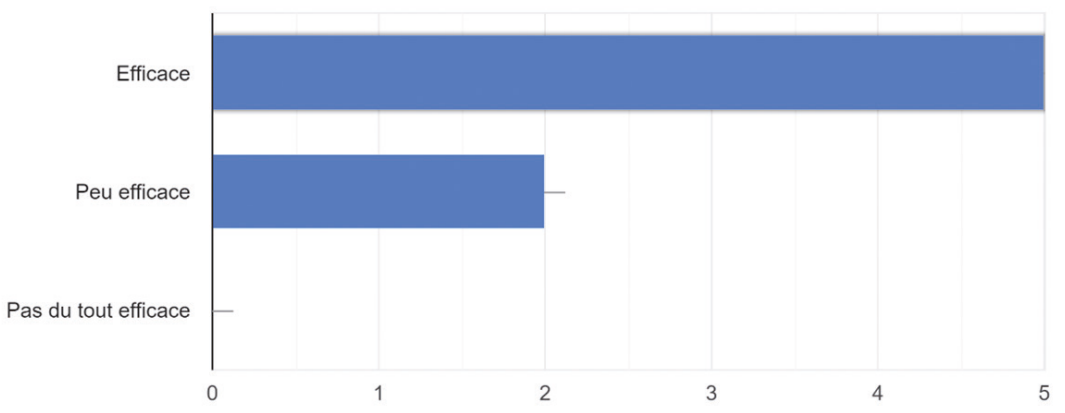


Anales de Filología Francesa, n. ${ }^{\circ}$ 29, 2021

ENSEIGNER LES COMPÉTENCES INTERCULTURELLES À L'ÈRE DU COVID 19, EXPÉRIENCE E-TANDEMS...

Avez-vous rencontré des problèmes à travailler en ligne?

8\&nbsp;réponses

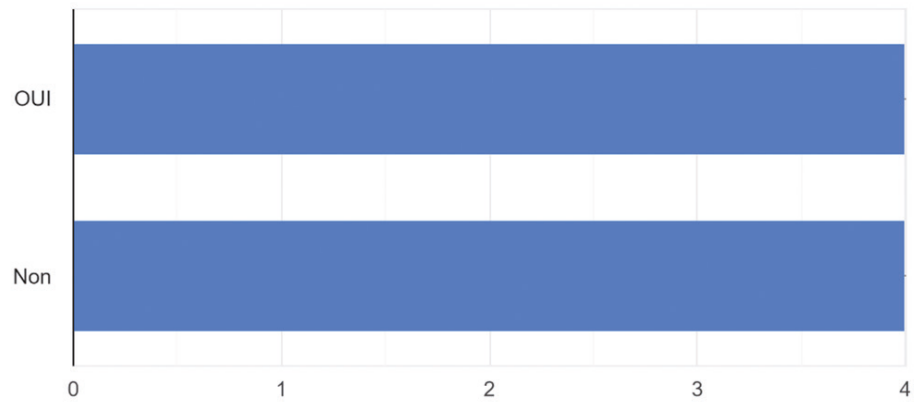

Quels outils numérique avez-vous utilisé?

8\&nbsp;réponses

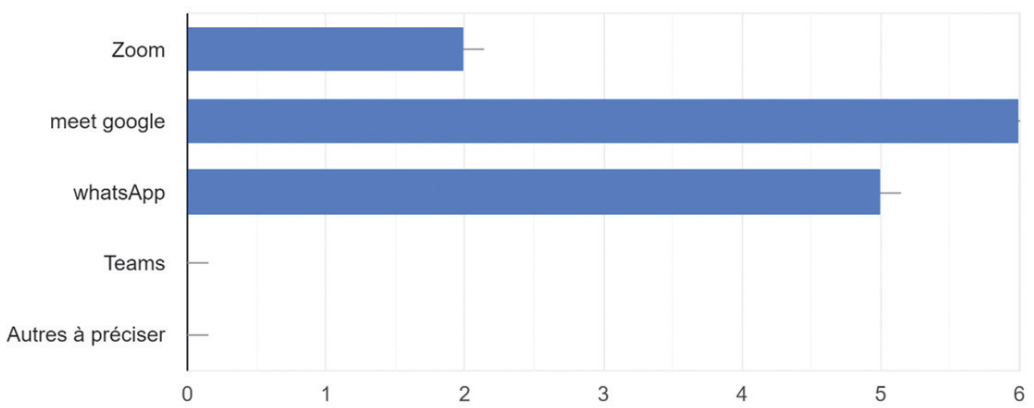


Quelle est la dimension qui vous a interpellé dans cette expériences E-Tandem? 8\&nbsp;réponses

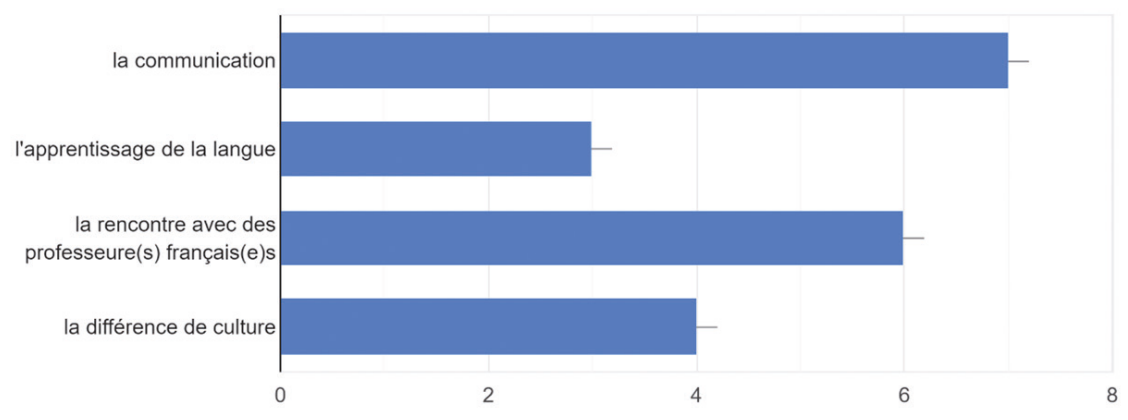

Qu'est-ce que vous appris de cette expérience au niveau culturel? 5 réponses:

o Même en parlant la même langue qui est le Français, il y'a toujours une différence entre les deux culture Française et Marocaine, en donnant l'exemple de la manière de communication selon le contexte, les termes utilisés par les individus, ou bien le comportement de chacun envers les autres.

o La différence de culture et les échanges qu'on a pu faire grâce à cette dernière

o j ai appris des nouvelles informations que je ne savais pas concernant la culture française soit dans le domaine du sport ou bien de la politique ou bien de la musique

o La langue par la communication et des informations sur l'État français

o Beaucoup

Pensez-vous que cette expérience est à

8\&nbsp;réponses

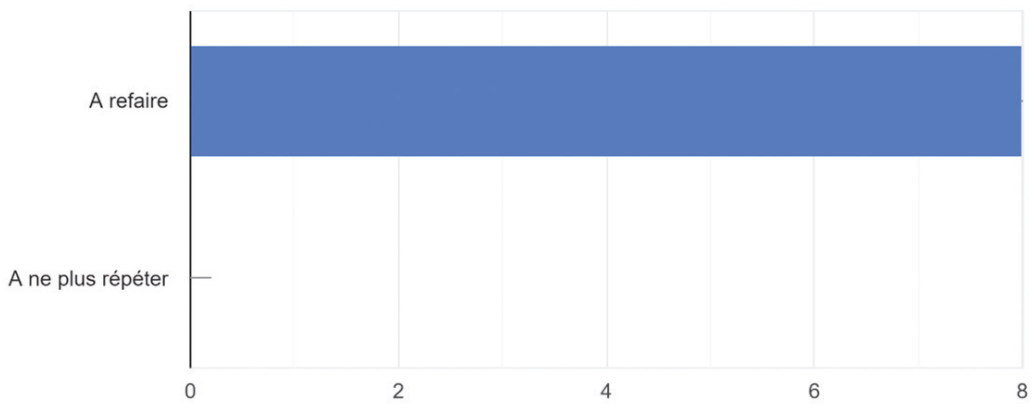


